\title{
Distances of absorbing features in the LDN 1622 direction
}

\section{An application of Tycho-2 photometry and Michigan Classification}

\author{
J. Knude ${ }^{1}$, C. Fabricius ${ }^{1}$, E. Høg ${ }^{1}$, and V. Makarov ${ }^{2, \star}$ \\ 1 Niels Bohr Institute for Astronomy, Geophysics and Physics, Juliane Maries Vej 30, 2100 København Ø, Danmark \\ 2 Universities Space Research Association, 300 D St. SW, Washington, DC 20024, USA \\ Received 13 December 2001 / Accepted 27 June 2002
}

\begin{abstract}
With Tycho- $2 B_{\mathrm{T}}$ and $V_{\mathrm{T}}$ photometry of Hipparcos stars with $\pi>8.0$ mas, $\sigma_{\pi} / \pi<15 \%$ and with spectral type and luminosity classification from the Michigan Catalogues we have a presumably unreddened sample for establishing a $\left(B_{\mathrm{T}}-V_{\mathrm{T}}\right)_{0}-$ $M_{V_{\mathrm{T}}}$ main sequence relation in the range from A0 to G5. We discuss the location of the median main sequence relation relative to published ZAMS relations for the A0-G5 range in some detail since the applications of this relation will have to assume that the evolutionary status of the calibration sample is representative of the local disk. If the ZAMS is defined as the lower envelope of the class V stars the published versions we discuss seem to be too bright by about half a magnitude in the F0-G5 range. The resulting intrinsic color and absolute magnitude estimates have errors of the mean of 0.03 and $0.3-0.4$ respectively. From this relation any given star with Tycho-2 photometry, spectral classification and luminosity class $\mathrm{V}$ can have its color excess $E\left(B_{\mathrm{T}}-V_{\mathrm{T}}\right)$ and distance modulus estimated. This means that the local interstellar extinction may be estimated for the $\sim 50 \%$ of the sky where Michigan classification presently is available. The individual color excesses and distance moduli may not be particularly accurate but we propose that they may be used to indicate extinction discontinuities caused by interstellar material at well defined distances. We have applied this new technique on stars from the Michigan Catalogue in the direction of $\operatorname{LDN} 1622(l, b)=\left(204^{\circ} .7,-11^{\circ} .8\right)$ supposed to be associated to the Orion B region 400-500 pc away but color excesses $E\left(B_{\mathrm{T}}-V_{\mathrm{T}}\right)$ exceeding 0.15 start appearing already in the distance slot 160-200 pc. Presently we cannot decide whether this nearby dust is related to LDN 1622 or whether this cloud is associated to Orion B. In a final comparison we study the color excess - distance variation in a $4^{\circ}$ region centered on LDN 1622 from Hipparcos - Tycho- 1 - Michigan data and the dust at $\sim 160 \mathrm{pc}$ is confirmed.
\end{abstract}

Key words. ISM: individual objects: LDN 1617, LDN 1622 - ISM: dust, extinction - ISM: cloud - techniques: photometric

\section{Introduction}

The distribution of the interstellar matter in the solar vicinity is of great interest since it may be related to phenomena on larger scales than just the dimension of individual clouds - the Local Bubble, the Gould Belt, the Vela - Gum region etc. The all sky surface distribution of atomic hydrogen is well known from the $21 \mathrm{~cm}$ data; the situation for the dust distribution is similar for the fraction having emission at $100 \mu \mathrm{m}$, and shows up in the sky maps (Schlegel et al. 1998). Both these distributions, however, are missing direct information on the matter's location in depth. For extinction maps based on photometry of individual stars each sight line provides an upper distance limit for the dust but the high density of sight lines needed for mapping often confines the analysis to small areas of special interest. The work by Vergely et al. (1998) combining the literature $u v b y \beta$ data and Hipparcos parallaxes may however give an indication of the local all sky dust distribution including upper distance

\footnotetext{
Send offprint requests to: J. Knude, e-mail: indus@astro.ku.dk

* Present address: USNO, 3450 Massachusetts Ave. NW, Washington, DC 20392-5420, USA
}

limits of the dust. But these input catalogues, photometric as well as astrometric, may contain serious biases making the dust census less systematic since they are made up of stars from many heterogeneous programs.

A new promising and more systematic option for identifying nearby extinction discontinuities seems to have become available. For the first time a homogeneous all sky photometric catalogue with a precision of a few hundredths of a magnitude has become available in the form of the $B_{\mathrm{T}}$ and $V_{\mathrm{T}}$ photometry in the Tycho-2 Catalogue (Høg et al. 2000). For $V_{\mathrm{T}}<9$. 0 the median standard error of $V_{\mathrm{T}}$ and $\left(B_{\mathrm{T}}-V_{\mathrm{T}}\right)$ is better than 0.014 and 0.025 respectively.

The two Tycho bands do of course not provide an intrinsic classification, for which one must rely on the Michigan spectral types and luminosity classes. The completeness and distance range is defined by the Michigan Catalogues since practically all stars in the Michigan Catalogues have Tycho-2 photometry. The faint limit of the Michigan classification defines the maximum color excess that may be observed at a given distance, see e.g. Fig. 5 of Nielsen et al. (2000) how this works for a much fainter sample than the Michigan stars. The upper 
distance limit for the Tycho-2 - Michigan combination is only a few hundred pc and $E\left(B_{\mathrm{T}}-V_{\mathrm{T}}\right)$ only a reaches few tenths of a magnitude. For the distance limit of Tycho- $2 \cap$ Michigan in the direction of LDN 1622 see Fig. 4 and the discussion of this issue in Sect. 3. We have used the recently published cross reference table between HD and Tycho-2, Fabricius et al. (2002) in the Tycho-2 - Michigan comparison.

The recently published 5th Michigan Volume has procured classification for the declination range from -12 to $+5^{\circ}$ (Houk \& Swift 1999) and the southern hemisphere is thus complete. To estimate color excesses and distances for HD stars in the southern half of the sky we only need to calibrate the 2-dimensional classification in terms of $\left(B_{\mathrm{T}}-V_{\mathrm{T}}\right)_{0}$ and $M_{V_{\mathrm{T}}}$.

In our particular context we try to estimate the distance to any nearby dust in an area containing the star forming molecular cloud LDN 1622, $\left[(l, b)=\left(204.7,-11^{\circ} 8\right),(\alpha, \delta)_{1950}=\right.$ $\left.\left(5^{\mathrm{h}} 52^{\mathrm{m}}, 1^{\circ} 47^{\prime}\right)\right]$ just a few degrees to the south of the new northern limit of the published Michigan classification. LDN 1622 is interesting because it is forming stars actively and because its location offers some means to test our present suggestion to estimate distances and reddening values.

The size of LDN 1622 is less than $1^{\circ} \times 1^{\circ} \quad$ (Fig. 1, Lee \& Myers 1999), but as Fig. 1 of Nakajima et al. (1998) shows, a larger region around LDN 1622 contains several $\mathrm{H}_{\alpha}$ emissionline stars supposed to be pre-main sequence stars. The extinction in a similarly wider area may therefore be of interest. A wide angular distribution of pre-main sequence stars may be caused by the velocity dispersion in a coeval group of stars, or could be the result of the star formation process propagating through a molecular cloud as could be the case in the nearby $\lambda$ Ori star forming region (Dolan \& Mathieu 2001). The last suggestion may be preferred if LDN 1622 is associated with Orion OB 1b. The wind swept appearance of the former cloud is indicative of an interaction with UV bright stars.

LDN 1622 is an example of a bright rimmed cloud and for some time we thought that there might have been a marginal, secondary option for the source of ionization etc. in HD 39572, formerly classified as B9. According to the Hipparcos Catalogues this star is located at $200 \mathrm{pc}$ where our first preliminary study of the Hipparcos/Tycho data also suggested the presence of reddening material. This option may, however, have been ruled out by now, since the classification of HD 39572 has been changed to A1mA5-A9 (Houk \& Swift 1999), a type hardly able to provide the required ionizing radiation. LDN 1622 contains optically identified cores (Lee \& Myers 1999) and other, different cores showing up in mm data (Jørgensen et al. in preparation). It is actively forming stars so its distance is essential for estimating timescales for outflows and masses of the dense cores and of the complete cloud. Its present distance estimate, 400-500 pc (see the compilation of LDN distances by Hilton \& Lahulla 1995), is based on an assumed proximity to the early type stars ionizing its edges facing the Orion $\mathrm{OB} 1 \mathrm{~b}$ association. The sight line to LDN 1622 is however located near the Gould Belt. At $l=205^{\circ}$ the nominal latitude of the Belt is $b=-23^{\circ} .4$ but as the Belt is about $20^{\circ}$ wide (Guillout et al. 1998) any dust detected in the $(l, b)=(204.7,-11.8)$ direction might be associated with the Belt. According to these authors, however, the Gould Belt encompasses the Orion nebulae at this longitude so an alternative association of the cloud with the Belt would not change the adopted distance of $\approx 400 \mathrm{pc}$. Another option for the dust we detect is an association to the Taurus complex of clouds with typical distances of $\approx 150 \mathrm{pc}$. The galactic latitude for LDN 1622 is similar to Taurus' and the longitude difference is $\approx 20-25^{\circ}$, thus at 150 pc LDN 1622 could be only $\approx 75$ pc from Taurus. Association of separate molecular material over similar, large distances is known for the Southern Coalsack Musca - Chameleon clouds (e.g. Corradi et al. 1998).

In a recent review of the life of molecular clouds (Bally 2001) Orion is taken as an example. LDN 1617, a neighboring cometary cloud, has a quite different velocity than LDN 1622 but the orientations of the two clouds are identical. Although they are both located on the outside of the main ionized feature, their surfaces facing Orion OB $1 \mathrm{~b}$ are ionized. One might speculate whether these clouds could be located on distinct sides of the features, expanding due to the early formation of massive stars (a total of $10^{3} \mathcal{M}_{\odot}$ is suggested in the form of 8-80 $\mathcal{M}_{\odot}$ mass stars). After Bally (2001) Orion is part of an even larger structure, the Cas-Tau super shell created by the Cas-Tau group, an $50 \mathrm{Myr}$ old fossil OB association. If the fragmentation of expanding shells may form molecular clouds, the distances to LDN 1617 and 1622 could be quite different and could deviate significantly from the distance to the massive stars in Orion OB $1 \mathrm{~b}(\approx 400 \mathrm{pc})$.

This paper proposes a method to estimate distances to nearby molecular material.

\section{2. $\left(B_{\mathrm{T}}-V_{\mathrm{T}}\right)_{0}$ and $M_{V_{\mathrm{T}}}$ calibration for luminosity class V stars}

Tycho-2 stars used for the calibration have Hipparcos parallaxes $\pi>8.0$ mas with $\sigma_{\pi} / \pi<15 \%$ and classification from the five published volumes of the Michigan Catalogue. The calibration sample comprises stars with 2-dimensional classification (spectral type and luminosity class V), Hipparcos parallaxes and Tycho-2 photometry for 7075 stars allocated to 31 spectral types in the range from A0 to G5. Due to the small upper distance limit of this sample it may be assumed to be unreddened, no reddening corrections are applied to the calibration sample.

Only stars in the range from A0 to G5 have been included in the calibration. There are too few nearby unreddened stars earlier than $\mathrm{A} 0$ to extend the calibration blue wards and in the region later than $\sim$ G5 misclassified evolved stars increase the scatter in $M_{V_{\mathrm{T}}}$.

The idea is to assign an intrincsic Tycho- 2 color and absolute magnitude to each of the 31 spectral types. The relative accuracy of $M_{V_{\mathrm{T}}}$ and the validity of the assumption $\left(B_{\mathrm{T}}-V_{\mathrm{T}}\right)_{0}=$ $\left(B_{\mathrm{T}}-V_{\mathrm{T}}\right)$ depend on the size of the parallax and on the parallax error but the parallax had to be lowered to a limit of $\approx 8.0$ mas in order to have enough stars of the rarer types such as A0/1 V (16 stars), A5 V (30 stars), A8/9 V (13 stars), G0/2 V (33 stars). The most frequent types are F5 V (815 stars), F7 V (561 stars), G3 V (620 stars) and G5 V (590 stars). The equivalent maximum distance of $125 \mathrm{pc}$ is however so large that some stars (presumably the early type ones) may be slightly reddened but we assume that the reddening of the calibration sample is 


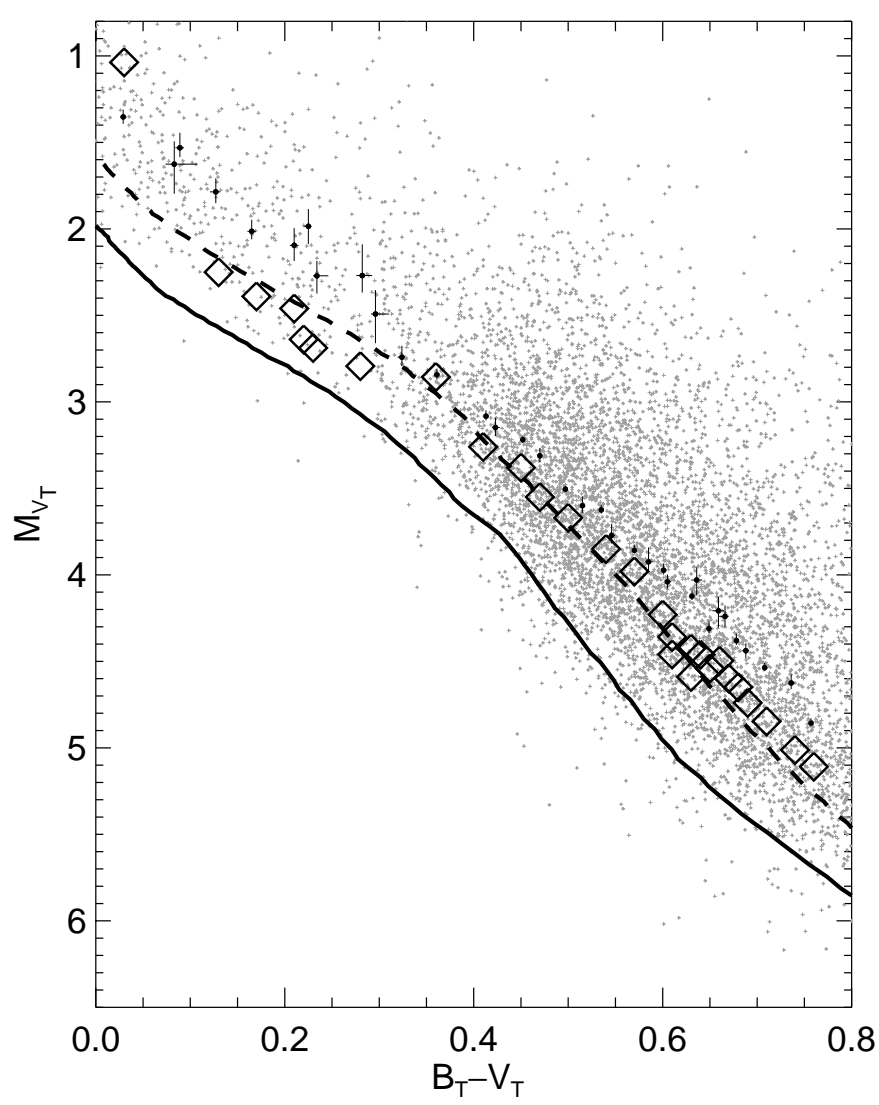

Fig. 1. Points with error bars display the resulting $M_{V_{\mathrm{T}}}$ vs. $\left(B_{\mathrm{T}}-V_{\mathrm{T}}\right)_{0}$ main sequence relation based on stars with $\pi>8.0$ mas and $\sigma_{\pi} / \pi<$ $15 \%$. Due to the rather short limiting distance of $125 \mathrm{pc}$ we may assume that $\left(B_{\mathrm{T}}-V_{\mathrm{T}}\right)_{\text {observed }}=\left(B_{\mathrm{T}}-V_{\mathrm{T}}\right)_{0}$. The spectral range covered includes the main sequence from A0 to G5. Error bars are errors of the median, estimated separately for each wing of the distribution from the 16 and $84 \%$ percentiles, see text. The diamonds are the ZAMS relations from Crawford (A and F stars) and Olsen (late F to G5 stars). The two curves are isochrones from Lejeune \& Schaerer (2001) both with an age $10^{3} \mathrm{yr}$, the upper one with $Z=0.020$ and the lower one with $Z=0.008$. The relations are plotted on the background of all Hipparcos, Tycho-2, Michigan stars fulfilling the selection criteria.

negligible. Presently we only calibrate stars with Michigan luminosity class $\mathrm{V}$ in terms of intrinsic Tycho- 2 color $\left(B_{\mathrm{T}}-V_{\mathrm{T}}\right)_{0}$ and absolute magnitude $M_{V_{\mathrm{T}}}$.

We keep the first part of our discussion in terms of Tycho-2 photometry instead of converting to the Johnson system: (B$V)_{\mathrm{J}} \approx 0.85(B-V)_{\mathrm{T}}$ and $V_{\mathrm{J}} \approx V_{\mathrm{T}}-0.090(B-V)_{\mathrm{T}}$ Eq. $(1.3 .20)$ in ESA (1997). A conversion may introduce additional uncertainty in color excess and distance estimates. We mention two problems of a main sequence calibration. First we represent the main sequence by a single luminosity calibration paying no attention to stellar evolution. The evolutionary stage of the relation is determined by the selection of Hipparcos stars for the calibration sample (see Fig. 1) and it may vary with the parallax limit chosen; there is little guarantee that the sample within 8.0 mas is representative of the galactic disk, but the calibration does not depend on whether all spectral types are included to the limiting distance which they are not. Only stars in the Hipparcos survey is complete to a common limiting magnitude, depending on the galactic latitude. The less luminous stars are only present within about 50 pc so unwanted reddening seems only to be a possible problem for the earliest spectral types. For what the effect of the variation of evolution may mean for an individual star see the discussion of HD 37443 in Sect. 3. The second problem is that the intrinsic colors and absolute magnitudes are affected by metallicity. We neglect this problem which may not be serious statistically, because most stars have the same solar-like metallicity. In general less than $10 \%$ have the thick disk and halo composition. Error bars of Fig. 1 include the scatter caused by these two omissions.

The absolute magnitude calibration may be affected by Malmquist bias but this correction turns out to be negligible. A conclusion resulting from an investigation of the size of the correction similar to that performed in Knude et al. (1999). The maximum correction $\lesssim 0 \mathrm{~m} 04$ is found for the late A type stars and not for the less luminous stars.

For stars with Tycho-2 photometry and measured spectral classification the color excess and absolute magnitude is immediately derivable from the spectral type $-\left(B_{\mathrm{T}}-V_{\mathrm{T}}\right)_{0}-$ $M_{V_{\mathrm{T}}}$ relation. For the distance moduli, $V_{\mathrm{T}}-M_{V_{\mathrm{T}}}-A_{V_{\mathrm{T}}}, A_{V_{\mathrm{T}}}$ is needed implying that the ratio $\frac{A_{V_{\mathrm{T}}}}{E\left(B_{\mathrm{T}}-V_{\mathrm{T}}\right)}$ must be known. We use a value 2.68 derived from Tycho- 2 photometry of two larger regions containing a wide range of extinctions, e.g. in Taurus (Knude \& Fabricius in preparation). The two regions we use are $\alpha_{2000}: 3^{\mathrm{h}} 0^{\mathrm{m}}-4^{\mathrm{h}} 4^{\mathrm{m}}, \delta_{2000}: 25^{\circ}-35^{\circ}$, and $\alpha_{2000}: 4^{\mathrm{h}} 0^{\mathrm{m}}-5^{\mathrm{h}} 0^{\mathrm{m}}, \delta_{2000}$ : $23.5^{\circ}-28.3^{\circ}$. $\left(B_{\mathrm{T}}-V_{\mathrm{T}}\right)-V_{\mathrm{T}}$ diagrams for such regions often display a linear confinement to the bright red side. We state that such a linear feature is caused by identical stars (probably clump giants) at approximately identical distances but with differing extinction. The slope of this confinement equals the reddening ratio $\frac{A_{V_{\mathrm{T}}}}{E\left(B_{\mathrm{T}}-V_{\mathrm{T}}\right)}$. And for both Taurus regions this slope is found to 2.68 for an extinction range of approximately four magnitudes. The large range makes the slope accurate. See Figs. 9 and 10 in Knude et al. (1999) for a published discussion of the reality of such common giant confinements. The suggested reddening ratio may be confirmed (or derived) from the Tycho-1 - Johnson transformation presented in the introduction to the Hipparcos-Tycho Catalogues, ESA (1997), and the canonical value of $R_{V}=\frac{A_{V}}{E(B-V)}=3.1$. The canonical value $R_{V}=3.05$ was recently confirmed in an extinction study of Taurus (Arce \& Goodman 1999), $R_{V_{\mathrm{T}}} \approx 0.85 \times\left(R_{V}+\frac{0.090}{0.85}\right)=$ 2.683 in good agreement with our suggestion for $R_{V_{\mathrm{T}}} ; 0.090$ and 0.85 are the coefficients in the magnitude and color transformations from the Tycho system to the Johnson system, see equations above.

The resulting color and magnitude calibration for the main sequence is presented in Table 1 in the form of median intrinsic color and median absolute magnitude for each spectral class. The 16 and $84 \%$ percentiles used for the error evaluation in the next paragraph are also given.

Figure 1 displays $\left(B_{\mathrm{T}}-V_{\mathrm{T}}\right) \equiv\left(B_{\mathrm{T}}-V_{\mathrm{T}}\right)_{0}$ and $M_{V_{\mathrm{T}}}$ median values for each stellar type of class $\mathrm{V}$ starting with A0 and ending with G5, error bars shown are derived from the 16 and $84 \%$ percentiles. Colors do show a symmetric distribution for each type but the absolute magnitudes are slightly skewed towards the faint part of their distribution; the mean of 
Table 1. Median intrinsic colors $\left(B_{\mathrm{T}}-V_{\mathrm{T}}\right)_{0}$ and absolute magnitudes $M_{V_{\mathrm{T}}}$ of luminosity class $\mathrm{V}$ stars. Based on Tycho-2 stars measured by Hipparcos and with $\pi>8$ mas and $\sigma_{\pi} / \pi<0.15$. 16 and $84 \%$ percentiles also given. Column 2 is the number of stars of a given classification.

\begin{tabular}{lrrccccc}
\hline \hline Type & $\#$ & $16 \%$ & $\left(B_{\mathrm{T}}-V_{\mathrm{T}}\right)_{0}$ & $84 \%$ & $16 \%$ & $M_{V_{\mathrm{T}}}$ & $84 \%$ \\
\hline A0 & 135 & -0.01 & 0.03 & 0.07 & 0.86 & 1.35 & 1.81 \\
A0/1 & 16 & 0.05 & 0.08 & 0.18 & 1.10 & 1.63 & 2.31 \\
A1 & 83 & 0.05 & 0.09 & 0.13 & 0.74 & 1.53 & 2.02 \\
A2 & 42 & 0.09 & 0.13 & 0.16 & 1.28 & 1.79 & 2.21 \\
A3 & 67 & 0.13 & 0.17 & 0.20 & 1.47 & 2.01 & 2.39 \\
A4 & 34 & 0.18 & 0.21 & 0.24 & 1.51 & 2.09 & 2.63 \\
A5 & 30 & 0.20 & 0.22 & 0.24 & 1.44 & 1.98 & 2.55 \\
A7 & 19 & 0.22 & 0.23 & 0.29 & 1.90 & 2.27 & 2.72 \\
A8 & 21 & 0.25 & 0.28 & 0.33 & 1.44 & 2.27 & 2.72 \\
A8/9 & 13 & 0.28 & 0.30 & 0.35 & 1.99 & 2.49 & 3.10 \\
A9 & 86 & 0.28 & 0.32 & 0.35 & 2.14 & 2.74 & 3.22 \\
F0 & 245 & 0.32 & 0.36 & 0.40 & 2.29 & 2.84 & 3.30 \\
F2 & 276 & 0.38 & 0.41 & 0.45 & 2.51 & 3.08 & 3.48 \\
F2/3 & 57 & 0.40 & 0.42 & 0.46 & 2.72 & 3.15 & 3.52 \\
F3 & 545 & 0.42 & 0.45 & 0.49 & 2.78 & 3.22 & 3.65 \\
F3/5 & 158 & 0.44 & 0.47 & 0.50 & 2.75 & 3.31 & 3.79 \\
F5 & 815 & 0.47 & 0.50 & 0.53 & 2.94 & 3.50 & 3.95 \\
F5/6 & 113 & 0.49 & 0.51 & 0.55 & 3.04 & 3.60 & 4.05 \\
F6 & 530 & 0.50 & 0.54 & 0.57 & 3.02 & 3.62 & 4.12 \\
F6/7 & 103 & 0.51 & 0.55 & 0.58 & 3.09 & 3.77 & 4.25 \\
F7 & 561 & 0.54 & 0.57 & 0.61 & 3.15 & 3.86 & 4.30 \\
F7/8 & 86 & 0.55 & 0.58 & 0.63 & 3.15 & 3.92 & 4.42 \\
F8 & 281 & 0.57 & 0.60 & 0.64 & 3.31 & 3.97 & 4.39 \\
F8/G0 & 133 & 0.58 & 0.61 & 0.65 & 3.57 & 4.04 & 4.50 \\
G0 & 460 & 0.59 & 0.63 & 0.67 & 3.44 & 4.12 & 4.59 \\
G0/1 & 39 & 0.60 & 0.64 & 0.67 & 3.57 & 4.03 & 4.59 \\
G0/2 & 33 & 0.62 & 0.66 & 0.69 & 3.75 & 4.21 & 4.76 \\
G1 & 260 & 0.60 & 0.65 & 0.69 & 3.63 & 4.31 & 4.73 \\
G1/2 & 75 & 0.61 & 0.67 & 0.70 & 3.69 & 4.24 & 4.76 \\
G2 & 336 & 0.64 & 0.68 & 0.72 & 3.65 & 4.38 & 4.83 \\
G2/3 & 114 & 0.65 & 0.69 & 0.73 & 3.99 & 4.44 & 4.90 \\
G3 & 620 & 0.66 & 0.71 & 0.75 & 3.85 & 4.54 & 4.99 \\
G3/5 & 152 & 0.70 & 0.74 & 0.78 & 4.02 & 4.63 & 5.07 \\
G5 & 590 & 0.71 & 0.76 & 0.81 & 4.18 & 4.86 & 5.29 \\
\hline & & & & & & &
\end{tabular}

the difference of the median absolute magnitude and the $16 \%$ percentile is $0.585 \pm 0.100$ and the mean difference between the $84 \%$ percentile and the median absolute magnitude is only $0.471 \pm 0.062$ (all stars included). If the two largest deviations are excluded, the $16 \%$ percentile mean difference is reduced to $0.571 \pm 0.088$. We may estimate the standard error by half the difference between the two percentiles implying that $\sigma\left(B_{\mathrm{T}}-V_{\mathrm{T}}\right)_{0}=0$. 035 and $\sigma\left(M_{V_{\mathrm{T}}}\right)=0$. 46 . The latter is an overestimate since it includes the main sequence width caused by evolution and varying metallicity. Since we may not estimate the effect of evolution from only two band photometry it should be left like it is, assuming that the Hipparcos calibration sample represents a fair sampling of unknown evolution across the main sequence present in the Michigan Catalogues. Taking these standard deviations as representative along the main sequence the relative distance error becomes $\approx 25 \%$, including photometric errors in $V_{\mathrm{T}}, M_{V_{\mathrm{T}}}$ and $A_{V_{\mathrm{T}}}$.

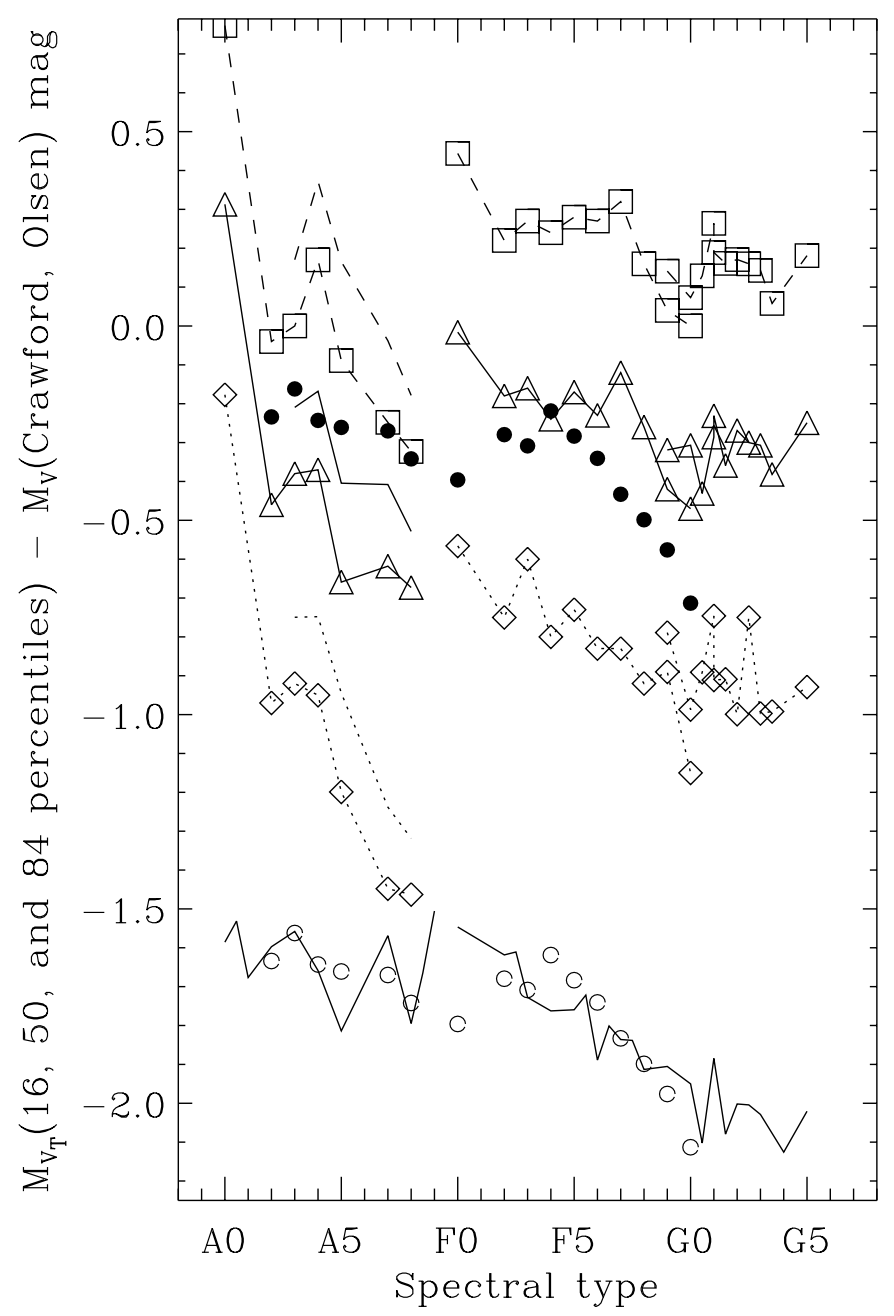

Fig. 2. Comparison of our $16 \%, 50 \%$ and $84 \% M_{V_{\mathrm{T}}}$ percentiles to Crawford and Olsens absolute Johnson magnitudes in the sense our $M_{V_{\mathrm{T}}}-M_{\text {Crawford,Olsen }}$ shown as a function of the Michigan spectral type classification. $\diamond: 16 \%$ percentile, $\triangle: 50 \%$ percentile (median), $\square: 84 \%$ percentile. The three curves without plotting symbols show the same comparison to Domingo \& Figueras (1999) for the late A-star range. The thick curve at the bottom indicates the comparison to our preliminary $\left(B_{\mathrm{T}}-V_{\mathrm{T}}\right)_{0}$ vs. $M_{V_{\mathrm{T}}}$ ZAMS relation shifted with $-1{ }^{\mathrm{m}} 0$, see Sect. 2.2. The filled circles are a comparison of a faint NGP sample to Crawfords A- and F-star standard relations. The open circles are the NGP comparison shifted $-1{ }^{\mathrm{m}} 4$.

\subsection{Comparison to ZAMS relations}

If the proposed Tycho-2 color - spectral type - magnitude relation does not represent the evolutionary state of a set of stars for which we want to determine distance moduli systematic deviations will be introduced.

We may evaluate the influence of stellar evolution from the proposed main sequence relation by comparing the deviations from some zero-age main sequence relation to the deviations of some similar, large unreddened sample's deviation from the same zero-age main sequence. Which ZAMS we compare to does not matter since we are only interested in the relative evolution from some standard curve in the HR-diagram. The comparison sample we use later in this section is an $u v b y \beta$ survey 
of all A3-G0 stars above $b=70^{\circ}$ with $B \leq 11^{\mathrm{m}} 5$, comprising 5400 stars (Knude unpublished).

In a series of papers (Crawford 1975, 1978, 1979; Olsen 1984) presented ZAMS relations for main sequence stars observed in the $u v b y \beta$ and $u v b y$ systems together with transformations between MK spectral types and $\beta$ and $(b-y)_{0}$ indices. Since $\beta$ and $(b-y)_{0}$ for class V stars are calibrated in terms of $M_{V}$ these indices may be used to establish a MK-type vs. $M_{V}$ (Crawford, Olsen) relation that can be compared to our Michigan type vs. $M_{V_{\mathrm{T}}}$ relation.

In the $u v b y \beta$ system $\beta$ is a measure of the strength of the Balmer line $\mathrm{H}_{\beta} . \beta$ is a good temperature indicator for $\mathrm{A}$ and $\mathrm{F}$ stars, hence the spectral type vs. $\beta$ relation. $\delta c_{0}=c_{0}-$ $c_{0}$ (ZAMS, $\beta$ ) where $c_{0}=(u-v)_{0}-(v-b)_{0}$. In the A-F star range $\delta \mathrm{c}_{0}$ is a measure of the evolution away from the ZAMS relation. $\delta m_{0}=m_{0}(\mathrm{ZAMS}, \beta)-m_{0}$ where $m_{0}=(v-b)_{0}-(b-y)_{0}$. In the A-F star range $\delta m_{0}$ is a measure of the metallicity $[\mathrm{Fe} / \mathrm{H}]$. $(b-y)_{0}$ is a reasonable temperature parameter for the $\mathrm{G} 0-\mathrm{G} 5$ range.

For the A0-G0 range we use the spectral type vs. $\beta$ relations given by Crawford and then his $\beta-M_{V}$ relation resulting in the spectral type $-M_{V}$ relation. Note that when we have the spectral type we also have the intrinsic Tycho- 2 color from the calibration. Similarly for the G0-G5 range where we use Olsen's spectral type $-(b-y)_{0}$ relation and then his $(b-y)_{0}-M_{V}$ relation, again resulting in the required spectral type $-M_{V}$ relation. We do not enter a discussion of the possible differences between the MK-classification used by Crawford and Olsen and the Michigan classification (deviations are probably only minor) but Olsen mentions that he made small adjustments to correct for different MK and Michigan classifications in the spectral range from G1 to G4. We do not discuss the differences between $V_{\text {Johnson }}$ and $V_{\text {Tycho }}$ either, apart from noting that the maximum systematic difference expected between F0 and G5 stars is less than 0.04 as indicated by $V_{\mathrm{J}}=$ $V_{\mathrm{T}}-0.090\left(B_{\mathrm{T}}-V_{\mathrm{T}}\right)$, ESA (1997). Crawford's and Olsen's ZAMS relations are shown as diamonds in Fig. 1. The luminosity comparison of the percentiles in the sense $M_{V_{\mathrm{T}}}(16 \%$, $50 \%, 84 \%)-M_{V}($ Crawford or Olsen $)$ is shown as $\diamond \mathrm{s}, \Delta \mathrm{s}$, and $\square$ s respectively in Fig. 2. Apart from the A0 stars all spectral types show the expected negative differences resulting from the evolution away from the ZAMS and some variation with spectral type is also noticeable. In particular the A stars show a large difference between the median and Crawford's ZAMS values. Crawford's calibration of the A stars is based on bright stars and a selection of clusters. Not on individual parallaxes. Since A stars may show large evolutionary effects we also show a comparison to a more recent calibration by Domingo \& Figueras (1999) based on the Hipparcos parallaxes. This paper states explicitly that a parallax limit $\pi>10$ mas and a relative accuracy $\sigma_{\pi} / \pi<20 \%$ were required for the calibration. Their resulting $M_{V}$ equation is linear in the $\beta$ index and for the comparison we have used Crawford's average $\beta$ values for the spectral types. We further assumed that $\delta c_{0}=0$ (no evolution) and that $\delta m_{0}=0$ (solar metallicity) and we paid no attention to the stellar rotation term $(v \sin i=0)$.

As the curves without plotting symbols in Fig. 2 show, the trend of the variation is as before but the differences are shifted by $\approx+0.20$ because the absolute magnitudes suggested by Domingo and Figueras are brighter than Crawford's. For A stars the color dependent $V_{\mathrm{J}}-V_{\mathrm{T}}$ variation is negligible compared to the shift of the absolute magnitude from A0 to F0.

Next we notice that there is a discontinuous shift between the A type stars and the F, G type stars approximately at the location of the Böhm-Vitense gap (for references see e.g. Newberg \& Yanny 1998). Due to the small number of stars in the gap (only $13 \mathrm{~A} 8 / 9$ stars), this is where the main sequence relation has the largest luminosity uncertainty as indicated by the error bars of Fig. 1. In fact, one of the reasons we relaxed the parallax limit to 8.0 mas was to include a significant number of stars in this particular region. The numerically largest deviations between the median main sequence relation (the $\triangle \mathrm{s}$ in Fig. 2) and Crawford's and Olsen's ZAMS relations are found for the A type stars. Despite their rather young ages this could be due to the fast evolution of the relatively more massive A stars. On the average the A stars in the calibration sample are more evolved that the bright stars and cluster stars used by Crawford. The shape of the Crawford A star ZAMS is defined by the lower envelope of a $c_{1}$ vs. $\beta$ diagram. Most local A stars from volume complete samples are younger than 1-2 Gyr with a median age of $\approx 0.8 \mathrm{Gyr}$ (Knude 1997; Chereul et al. 1999). The mean age of the six clusters used by Crawford is only 0.35 Gyr (Allen 2000).

In the range from F0 to G5 the differences also display a negative trend with decreasing temperature. The shift is most clearly seen for the $16 \%$ percentile and the median. In both cases the difference decreases with $0.0-0.5$ from F0 to G5. This is the expected trend of evolution mentioned above: the median absolute magnitude of the early $\mathrm{F}$ type stars is about as bright as the ZAMS but the early $\mathrm{G}$ type stars are about half a magnitude brighter than the ZAMS. Our particular sample of F0-G5 stars thus shows a larger degree of evolution with decreasing temperature. This reflects the effect of increasing ages from A $\sim 2$ Gyr for the early F type stars to A $\sim 10-15$ Gyr for the G5 stars. A different age mixture along the main sequence relation is no problem as long as the sample on which we apply the calibration has the same stage of evolution as the calibration sample.

\subsection{Is the evolutionary stage of the calibration sample typical?}

To investigate this issue a little further we have established a spectral type $-M_{V}$ relation for the NGP sample of A3-G0 stars mentioned above. We use the spectral type vs. $\beta$ tables given by Crawford. For a \pm 0.005 interval centered on $\beta$ for each of the tabulated spectral types we extract the relevant stars and compute the mean value of $c_{1}$. Since the latitude exceeds $70^{\circ}$ for the NGP sample these stars have a reddening that is negligible for most purposes and we may calculate $\delta c_{0}$ directly from the observations for each of the mean $c_{1}$ values corresponding to the spectral types. The magnitude change resulting from the evolution away from the ZAMS is given by $\Delta M_{V}=-f \times \delta c_{0}$ where $f=9.0$ for the A stars and $f=9.0+20 \times(2.720-\beta)$ in the $F$ range. The filled circles in Fig. 2 show the evolutionary status 
of the NGP sample as compared to Crawfords A and F ZAMS relations.

A comparison of the calibration sample to the NGP sample indicates some interesting differences in the $\mathrm{A}$ and $\mathrm{F}$ star ranges. In the A star interval the calibration sample appears somewhat more evolved than the NGP sample. Note that the NGP A stars are volume complete to $Z \approx 400$ pc (Knude 1997). In the F star range the calibration sample is less evolved than the NGP sample. Apparently the calibration sample consists of relatively older A stars and relatively younger F stars.

The evolutionary difference between the two samples are only some tenths of a magnitude $-0.2--0.4$ for the A stars and $+0 \mathrm{~m} .1-+0 \mathrm{~m} 2$ for the $\mathrm{F}$ stars (excluding F0). This means that if we had applied the calibration on the NGP sample the A star distances would have been increased by $5-10 \%$ and the $\mathrm{F}$ star distances would have been reduced by $2-5 \%$ compared to a calibration based on a main sequence relation similar to that of the NGP sample itself.

Apparently the calibration sample is not quite typical.

\subsection{Need for a modified ZAMS in the FO-G5 range}

In Fig. 1 Crawfords A star calibration seems to follow the lower envelope of the calibration data rather well and the suggested shift of the relation $\approx+0$. 2 (Domingo \& Figueras 1999) to the bright side may not be required. In the F-G5 spectral range the ZAMS relations by Crawford and Olsen are significantly brighter than the lower envelope of Fig. 1 and since it is customary to define the ZAMS as the faint envelope of the main sequence a revision might be appropriate. Presently we just approximate the lower envelope with two straight lines, $M_{V_{\mathrm{T}}}=4.517\left(B_{\mathrm{T}}-V_{\mathrm{T}}\right)+1.800$ for the A0-F0 range and $M_{V_{\mathrm{T}}}=$ $6.235\left(B_{\mathrm{T}}-V_{\mathrm{T}}\right)+1.142$ for the $\mathrm{F} 0-\mathrm{G} 5$ range. The difference between our main sequence relation and these simple ZAMS approximations is shown as the solid curve at the bottom of Fig. 2 (for clarity shifted -1.0 ). It is noticed that the discontinuity between the $\mathrm{A}$ and $\mathrm{F}$ stars has disappeared. The $\mathrm{A}$ star range now has a rather constant offset $-0.6 \pm 0.1$ and in the F-G5 star range the difference grows to numerically larger values towards the cooler stars, much as to be expected since the median ages grow with decreasing temperature.

A shift to a fainter absolute magnitude for a given color can be caused by shifting the metallicity from the disk values we tacitly assumed to be valid to the values typical for the halo, e.g. Jordi et al. (1997). It would, however, be somewhat strange if the least luminous stars common to the Hipparcos, Tycho-2, and Michigan catalogues were all metal poor. We may estimate whether the difference between the Crawford-Olsen ZAMS and the straight line approximation to the lower envelope we suggested above is significant. For this we may use the percentiles. The difference between the main sequence relation, established as the median of the $M_{V_{\mathrm{T}}}$ distribution for a given color (spectral type), and the Crawford-Olsen ZAMS is roughly 0.5 standard deviation. The difference between the CrawfordOlsen ZAMS and the lower envelope in the F0-G5 range is about 1.0 standard deviation. The standard deviation was estimated from the $16 \%$ and $84 \%$ percentiles discussed above.
We may then calculate the expected number of stars below the lower envelope from the number of stars in our sample in the F0-G5 range. About 400 stars are expected and about 200 are counted. On the other hand about 2500 stars out of 6500 in the F0-G5 sample are located below the Crawford-Olsen ZAMS so it may hardly be termed a lower envelope to the main sequence in the HR diagram.

The observational ZAMS of Crawford and Olsen compares rather well to theoretical isochrones. In Fig. 1 the dashed curve shows a $10^{3} \mathrm{yr}$ isochrone with solar metallicity $Z=0.02$ from Lejeune $\&$ Schaerer (2001). The $(B-V)$ colors and $M_{V}$ data pertaining to the isochrone are transformed to Tycho-2 colors and magnitudes as indicated in the begining of this section. For colors redder than $\left(B_{\mathrm{T}}-V_{\mathrm{T}}\right)_{0} \approx 0.65$ the observed relation is brighter by about 0.2 and in the late A-star color range from 0.2 to 0.3 the observed relation is low by about 0.3 . But the change of slope at $\left(B_{\mathrm{T}}-V_{\mathrm{T}}\right)_{0} \sim 0.3$ is similar for the observed and theoretical relations. Figure 1 also depicts a $10^{3} \mathrm{yr}$ isochrone with $Z=0.008$ that is systematically fainter than the sample's lower envelope for the $\mathrm{F}$ and $\mathrm{G}$ stars but otherwise follows the confinement until $\left(B_{\mathrm{T}}-V_{\mathrm{T}}\right)_{0} \approx 0.3$ where it runs significantly below the data. The suggested $\left(B_{\mathrm{T}}-V_{\mathrm{T}}\right)_{0}-M_{V}$ main sequence relation deviates least from the theoretical solar metallicity ZAMS in the color range $0.4-0.5$ that is in the F2-F5 type range according to Table 1. The largest deviations are found for the A- and G-type stars and are caused by fast evolution and old age respectively.

We notice a somewhat peculiar coincidence shown nethermost in Fig. 2: the shape of the difference between our main sequence relation and the lower envelope approximated by two straight lines is almost identical to the shape of the difference between the NGP main sequence and Crawford's ZAMS but there is a zero point shift of 0.4 ?

\section{Absorption features and their distance in the LDN 1622 direction}

An advantage using Michigan and Tycho-2 instead of Hipparcos and Tycho-1 is the larger number of stars per square degree. At the position of LDN 1622 Michigan provides about $35 \%$ more class V stars than Hipparcos despite we use a narrower spectral range from Michigan than from Hipparcos: A0-G5 vs. O-K7.

So if a star is measured by Tycho- 2 and have Michigan 2-dimensional classification we may use the median spectral type vs. $\left(B_{\mathrm{T}}-V_{\mathrm{T}}\right)_{0}$ and spectral type vs. $M_{V_{\mathrm{T}}}$ relation to derive an estimate of the color excess and the distance modulus for this star.

Due to the small size of LDN 1622 we may not expect to have many Michigan stars projected within its contours and we must rely on its being part of a more extended structure. This could in fact be the case as the color excess map in Fig. 9 shows. Figures 3 and 4 depict stars sampled inside 2.4 and 5.0 circles centered on the nominal position $(l, b)=\left(204^{\circ} .7\right.$, $-11.8)$. These two figures show the color excess $E\left(B_{\mathrm{T}}-V_{\mathrm{T}}\right)$ vs. $V_{\mathrm{T}}-M_{V_{\mathrm{T}}}-A_{V_{\mathrm{T}}}=5 \log r-5$ with $r$ in pc. The distance modulus $V_{\mathrm{T}}-M_{V_{\mathrm{T}}}-A_{V_{\mathrm{T}}}$ is quite a common way to present photometrically derived distances and it is a combination of a 


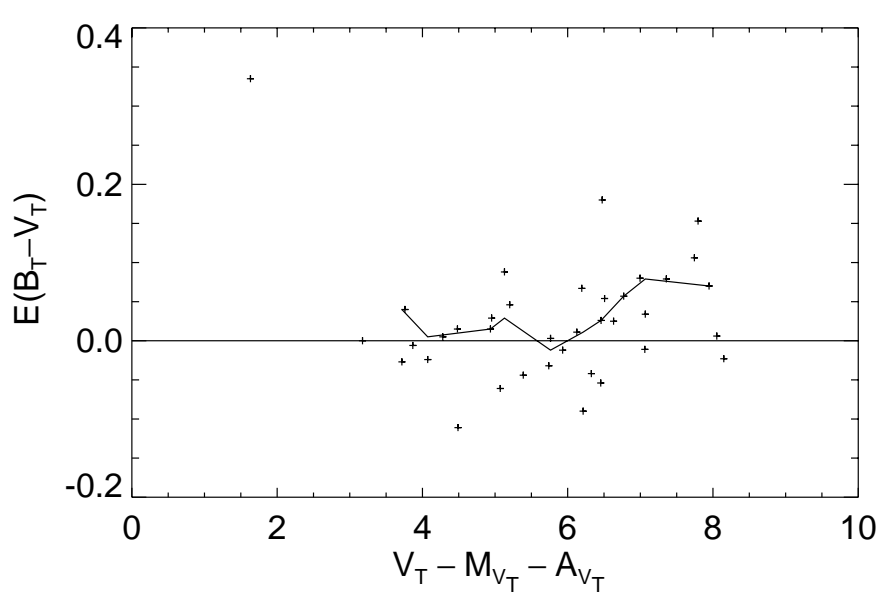

Fig. 3. Reddening vs. distance diagram derived from Tycho- 2 photometry and the Michigan calibration in Fig. 1. Stars within 2.4 from the nominal center of LDN 1622 are shown. Solid curve indicates discrete median values of $E\left(B_{\mathrm{T}}-V_{\mathrm{T}}\right)$ in 0.5 bins of the distance modulus $V_{\mathrm{T}}-M_{V_{\mathrm{T}}}-A_{V_{\mathrm{T}}}$.

measured parameter and quantities derived from the calibration. The median variation of the color excess with distance modulus is shown by the solid curve. An increase by almost $3 \times \sigma\left(B_{\mathrm{T}}-V_{\mathrm{T}}\right)$ above 0 in the color excess is noticed for the distance modulus in the range from 6.0 to 6.5. Generally, the excess exceeds $\approx 0$. 1 beyond this distance but a few sight lines with no reddening are also present. Absorbing material seems to be present within $160-200 \mathrm{pc}$ in the LDN 1622 direction. If we consider a region four times larger, the corresponding radius goes beyond the northern limit of the Michigan Catalogue and we get a less clear picture. Figure 4 shows the behavior of color excess with distance modulus. There is a marked difference in the excess distribution below and above $V_{\mathrm{T}}-M_{V_{\mathrm{T}}}-A_{V_{\mathrm{T}}}=6.3$, $r=180 \mathrm{pc}$. For the sample with distance modulus smaller than 6.0 mag the mean color excess is -0.001 with a scatter 0.042 if we leave out the six values more than $3 \times \sigma\left(E\left(B_{\mathrm{T}}-V_{\mathrm{T}}\right)\right)$ from zero reddening. At $180 \mathrm{pc} A_{V_{\mathrm{T}}} \lesssim 0$. 6 is encountered but again several sight lines penetrate equally far and show negligible reddening. Since about 4 stars show the extinction increase at $180 \mathrm{pc}$ the error of the mean is reduced to $13 \%$ implying a distance of $180 \pm 23 \mathrm{pc}$.

We note that the sample of Fig. 4 terminates abruptly at $V_{\mathrm{T}}-M_{V_{\mathrm{T}}}-A_{V_{\mathrm{T}}} \approx 8$ for $\left(B_{\mathrm{T}}-V_{\mathrm{T}}\right)-\left(B_{\mathrm{T}}-V_{\mathrm{T}}\right)_{0} \approx 0$ and that the most reddened stars at the distance confinement are located at $V_{\mathrm{T}}-M_{V_{\mathrm{T}}}-A_{V_{\mathrm{T}}} \approx 7.5$. The curved shape of this confinement is due to the combination of reddening and the limiting magnitude of our Tycho-2 $\cap$ Michigan sample and the fact that we have only included stars intrinsically fainter than $M_{V_{\mathrm{T}}} \approx 1$. 35 in our luminosity calibration. The reason for this bright limit is that too few stars of earlier type than $\sim \mathrm{A} 0$ are present within $\pi>8.0$ mas and with $\sigma_{\pi} / \pi<15 \%$ for a reliable calibration. In a comparison of two catalogues, the limiting magnitude is determined by the catalogue with the brightest limit, which in our case is the Michigan Catalogue(s). For the area confined by a radius equal to $5^{\circ}$, the $V_{\mathrm{T}}$ histogram for the Tycho-2กMichigan sample shows that the limiting magnitude is $V_{\mathrm{T}}=9 \mathrm{~m} .4$. The bright $M_{V_{\mathrm{T}}}=1.35$ limit and the faint $V_{\mathrm{T}}=9.4$ limit for
$A_{V_{\mathrm{T}}}=0$ implies $V_{\mathrm{T}}-M_{V_{\mathrm{T}}}-A_{V_{\mathrm{T}}}=8$ as is the case for the data in Fig. 4. The Tycho-2 $\mathrm{n}$ Michigan sample accordingly has an upper distance limit of $400 \mathrm{pc}$ in the LDN 1622 direction. This implies that the Tycho-2กMichigan sample just reaches out to the ionizing stars in the Orion OB $1 \mathrm{~b}$ association if the stars are unreddened. Reddened stars fall short due to the sampling of the two catalogues and to the limited spectral range of the absolute magnitude calibration. The stars in Fig. 4 with $A_{V_{\mathrm{T}}} \sim 0.5$ are only observed to a distance modulus $\sim 7.4$ corresponding to $\sim 300 \mathrm{pc}$.

Four apparently nearby stars seem to be very reddened in Fig. 4 having excesses above 0.15 and being closer than distance modulus $5.0(\sim 100 \mathrm{pc})$. Sorted according to the suggested distance modulus they are HD 40726, 39834, 37443 and 42112. HD 40726 is classified as G5V but its uvby photometry (Hauck \& Mermilliod 1998) indicates that it may be a giant of luminosity class III. Assuming that it is a G5III type it has $M_{V}=0.9 \pm 0.3$. The error estimate is relaxed. With the changed classification the distance modulus is shifted to the range $5.8-6.4$ and its color excess to $\approx 0$. 03 . There are no uvby photometry of HD 39834 and 42112. HD 39834 is however part of a suspected double star system and in SIMBAD it is classified as G5III not as G5V as in Michigan. If it is a giant and the error on the giant luminosity again is assumed to be $0.9 \pm 0.3$ its distance modulus will be in the range 6.1-6.7 instead of 2.4, again consistent with that of the extinction discontinuity but the color excess is now reduced to 0.08 . The $2 \mathrm{MASS}$ data for this star (Skrutskie et al. 1997) indicates no reddening neither if the star is class $\mathrm{V}$ nor if it is class III. In the $H-K$ vs. $J-H$ diagram it is located where the main sequence and giant relation coincide, Bessell \& Brett (1988). We have found no additional information on the classification of HD 42112 and its 2MASS data indicate no reddening. HD 37443 is also in the uvby compilation of Hauck and Mermilliod. According to this photometry it is not a luminosity class III star but is evolved $\Delta M_{V} \approx 1.4$ above Crawfords ZAMS relation. It is classified F2V, a region where our median relation and Crawfords relation run quite close, Table 1 and Fig. 1 . The intrinsic color may not have to be changed but $M_{V_{\mathrm{T}}}$ must be brightened by $\approx 1.5$. This means that the distance modulus should be shifted from 4.3 to $4.3+1.5=5.8$ and that the color excess remains unchanged. In Fig. 4 HD 37443 will move close to the extinction rise. That HD 37443 is reddened is also indicated by its 2 MASS photometry. It is situated well to the reddened side of the main sequence relation in the $H-K$ vs. $J-H$ diagram. HD 37443 is an example of the worst case distance error that the width of the main sequence may cause: the class V classification seems right but it has evolved to the upper part of the main sequence band. Fortunately such stars are statistically infrequent.

\section{1. uvby $\beta$ and $\mathrm{Nal}$ data}

The presence of dust at $d \lesssim 160$ pc may be corroborated by color excesses and photometric distances computed from $u v b y \beta$ photometry. We searched the compilation by Hauck \& Mermilliod (1998) for stars within 2.4 from the center of LDN 1622. 11 entries had photometry meeting the criteria for 


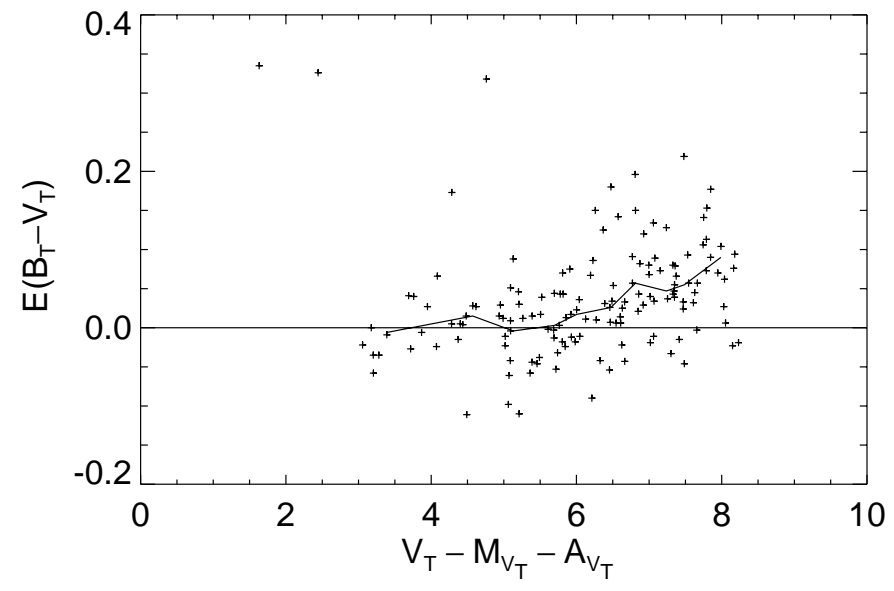

Fig. 4. Reddening vs. distance diagram derived from Tycho-2 photometry and the Michigan calibration in Fig. 1. Stars within 5.0 from the nominal center of LDN 1622 are included. Solid curve indicates discrete median values. The four deviant stars with color excess larger than 0.15 and distance modulus less than 5.0 are discussed in the text. Sorted according to distance modulus they are HD 40726, 39834, $37443,42112$.

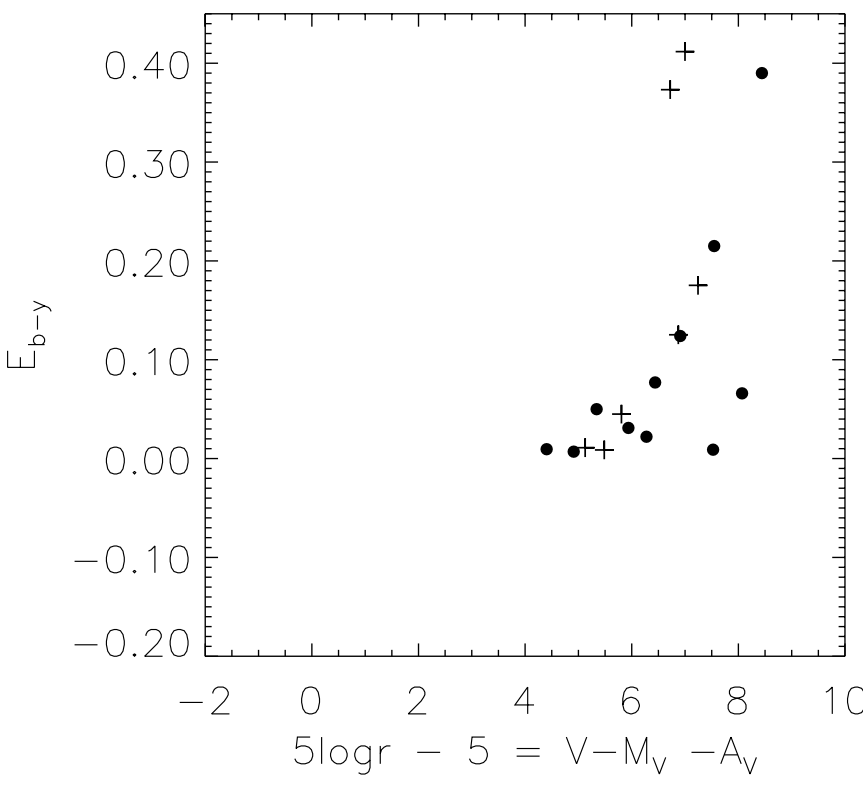

Fig. 5. Reddening from $u v b y \beta$ photometry within 2.4 from LDN 1622's nominal center. Abscissae 6.5 and 7.0 correspond to distances 200 and $250 \mathrm{pc}$ respectively. The plus signs are equivalent widths of the $\mathrm{NaI} \mathrm{D}_{2}$ lines of nearby early type stars in $\mathrm{m} \AA$ scaled down with a factor 400 from Sfeir et al. (1999).

computing intrinsic stellar properties. The resulting color excesses and photometric distance moduli are shown in Fig. 5. Note that these distances are from photometry alone despite parallaxes are available for a few stars. We notice the general increase of $E(b-y)$ with distance and in particular that two stars with modulus 6.5 and 7.0 have $E(b-y)=0.08$ and 0.12 respectively in concord with Figs. 3 and 4.

Two lines of evidence suggest that $A_{V} \lesssim 0.5$ may be present at only $\$ 200 \mathrm{pc}$ in the direction $(l, b)=(204.7,-11.8)$. But as it becomes evident in the next section we cannot claim that LDN 1622 has a distance between 160 and 200 pc, 400$500 \mathrm{pc}$ is certainly still an option.

From the recent mapping of the local NaI D absorption (Sfeir et al. 1999) we display the lines of sight near LDN 1622 as plus signs in Fig. 5. The angular separations from LDN 1622 are quite large, between 5 and 14 degrees but fortunately the sodium stars have lower latitudes than the cloud so the lines of sight are located inside the main ionization contours of Orion. The measured equivalent widths are probably related to the shells enclosing the Orion OB associations or as Sfeir et al. suggest to the confinement of The Local Bubble. We note that quite large equivalent widths are observed for $V-M_{V}-A_{V}$ between 6 and 7, based on Hipparcos parallaxes. Unfortunately the $\mathrm{NaI}_{2}$ radial velocities have not been published. The velocities are needed for a comparison to the $\mathrm{mm}$ velocities observed by Jørgensen (2000) for a precise identification of LDN 1622's distance.

The NaI $\mathrm{D}_{2}$ absorption contours provided by Sfeir et al. (1999) show that the contours corresponding to equivalent widths 50 and $20 \mathrm{~m} \AA$ have distances 150 and $125 \mathrm{pc}$ respectivly when projected onto the Galactic plane for longitude $205^{\circ}$. Within the errors this is compatible with the dust we find at $160 \mathrm{pc}$ in the $(205,-12)$ direction.

There is a further complication in assigning the extinction discontinuity to LDN 1622 owing to the fact that another cloud LDN $1617,(l, b)=(203.5,-11.9)$ is less than one degree from LDN 1622 and is included in the angular zones with radii 2.4 and 5.0 we have searched for Tycho-2 $\cap$ Michigan stars. Therefore any distance proposed for LDN 1622 may equally well apply to LDN 1617. The distance to LDN 1617 is of some interest since it is proposed to host one of the finest specimens of Herbig Haro flows, HH 111 (Reipurth \& Olberg 1991).

We end this Section by claiming that the calibration of the Michigan classification shows that a color excess discontinuity rises for virtually nothing to $E\left(B_{\mathrm{T}}-V_{\mathrm{T}}\right) \gtrsim 0$. 2 at a distance modulus 6.0-6.5 and that this jump is confirmed by color excesses and photometric distances from $u v b y \beta$ photometry, and from $\mathrm{NaI} \mathrm{D}$ absorption lines in stars with Hipparcos parallaxes.

\subsection{Using Hipparcos stars with measured spectral classification}

As mentioned above we may also apply the Hipparcos parallaxes and Tycho-1 colors transformed to the Johnson system as given in the Hipparcos Catalogue together with the spectral and luminosity classification by Houk \& Swift (1999) and intrinsic colors from Schmidt-Kaler (1982), the way we proposed for the nearby molecular clouds (Knude \& Høg 1998). For the Hipparcos stars we may use a wider spectral range of class V stars than when we use the Michigan main sequence relation: for Hipparcos we use the full $\mathrm{O}-\mathrm{K}$ main sequence. This means that B stars are included, which was not the case for the Michigan stars. For a given apparent magnitude this implies that large distances may be probed. This is a main reason why Fig. 6 includes larger distance moduli than Fig. 4.

In Fig. 6 we display the variation of $E_{B-V}$ vs. $V-A_{V}-M_{V}$ derived from stars in The Hipparcos Catalogue and in vol. 5 


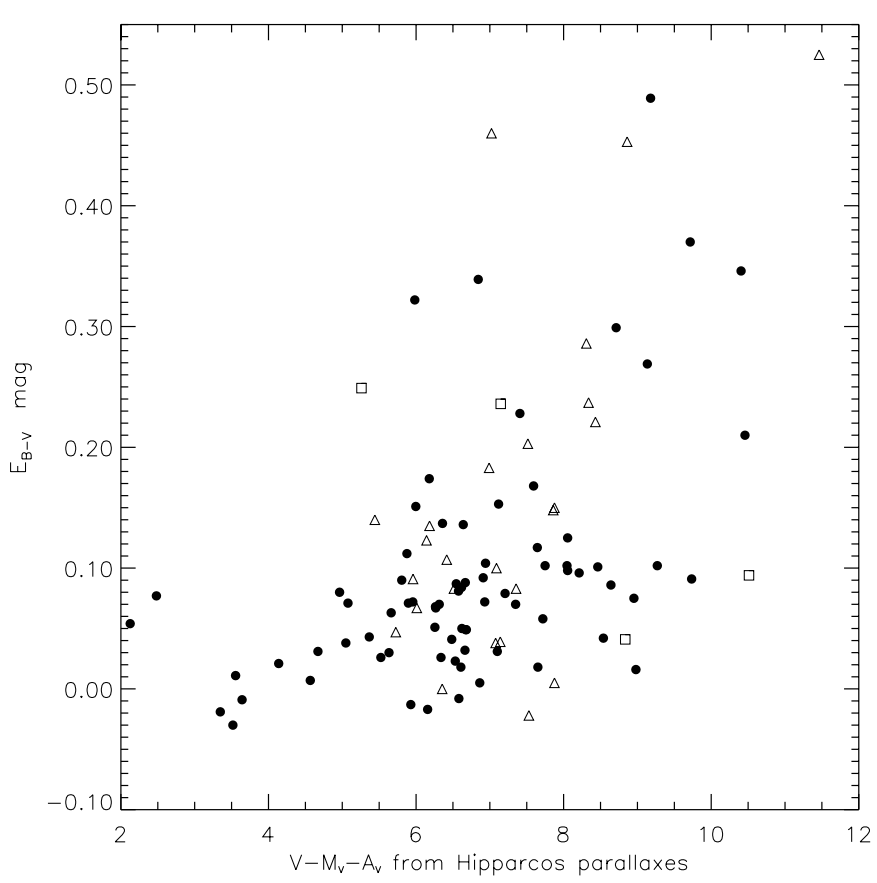

Fig. 6. Another way of estimating the interstellar structure function is by combining parallaxes, transformed Tycho- 1 photometry and Michigan spectral and luminosity classification. Dots are class V and triangles class III stars and the boxes are the four class II stars. The area covered by this diagram is circular with a radius of 4.0 centered on LDN 1622. On the average there are 2 lines of sight per square degree.

of the Michigan survey. The extent of LDN 1622 itself is rather small, according to Jørgensen et al. op.cit., only about 0.5 along its largest axis. Figure 3 of Bally (2001) shows a slightly larger size in the SW-NE direction, 50'. In order to have a continous coverage of the spectral range and to have enough Hipparcos stars we have to sample a larger area. Figure 6 is for stars within 4.0 from the nominal center of LDN 1622 . We only display the results from class II (4 stars), III (26 stars) and V (76 stars) despite a few stars of other luminosity classes are present. These approximately 100 stars allow a detailed study of the extinction distribution with about 2 lines of sight per square degree on the average.

We note that the color excess distribution has a discontinuity at $V-A_{V}-M_{V} \approx 6$ corresponding to a distance of $\sim 160 \mathrm{pc}$ or approximately $\pi=6$ mas. The $A_{V}$ values in the discontinuity reach above 0.5 and a single star have $A_{V} \sim 1$ mag. Considering that the typical parallax error of The Hipparcos Catalogue is about 1 mas this distance seems fairly well determined being based on $\sim 10$ stars defining the discontinuity.

Taken at face value one might claim that the distance to some molecular cloud seems to be $160 \mathrm{pc}$, much as the standard distance estimated to any of the nearest star forming clouds. But a four degree area is large for a small feature like LDN 1622. Recall that this solid angle also contains LDN 1617. It is not known if the color excesses are caused by a coherent structure. With an average of two stars per square degree the Hipparcos/Michigan sample may provide some information on the projected dust distribution. Figure 9 shows

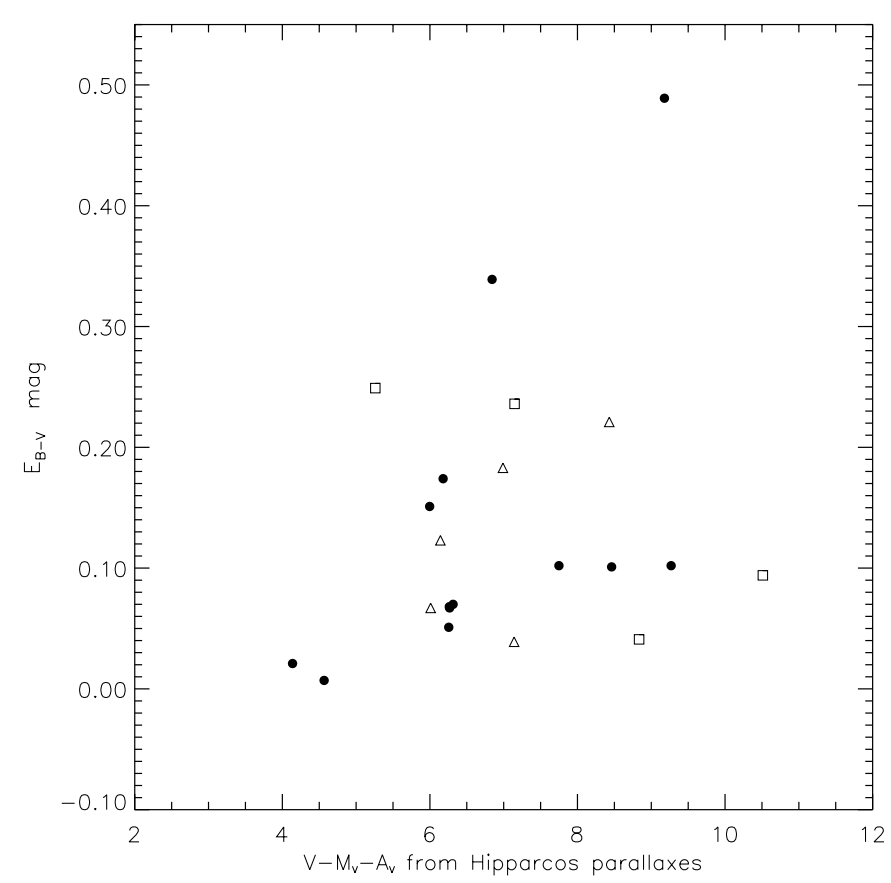

Fig. 7. Symbols as in Fig. 6. The area covered by this diagram is an annulus with radii of 1.0 and 2.0 respectively centered on LDN 1622.

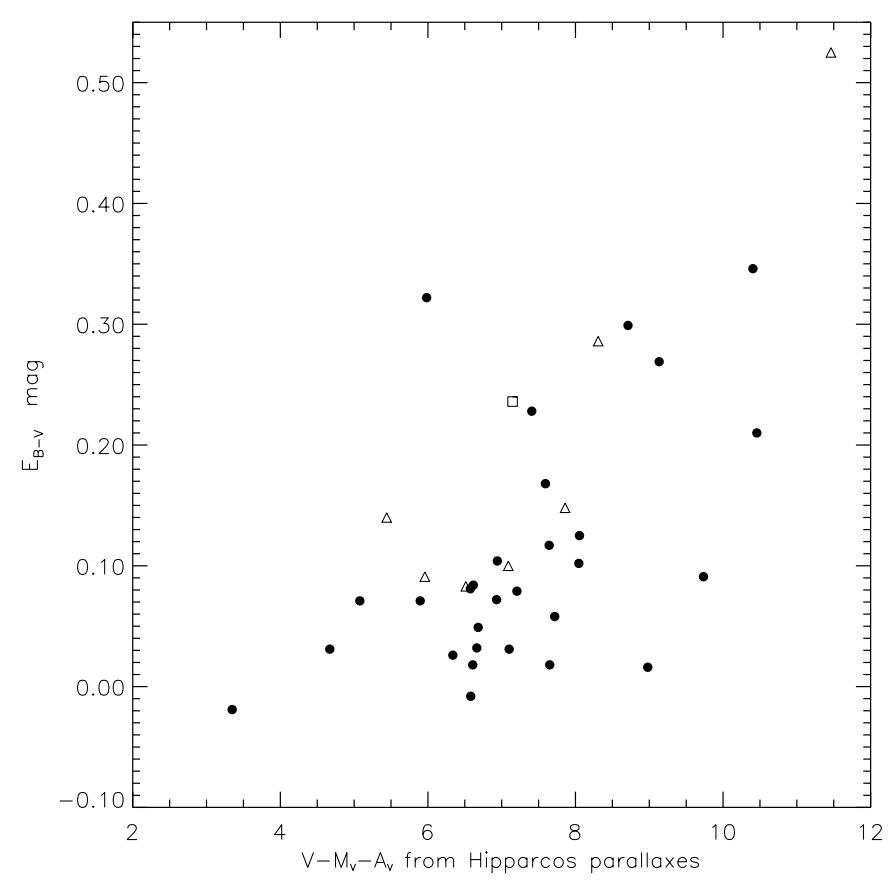

Fig. 8. Dots are class V and triangles class III stars whereas class II stars are depicted as small squares. The area covered by this diagram is circular ring with radii of 2.0 and 3.0 respectively centered on LDN 1622.

contours of the average $E_{B-V}$ formed for $1 \times 1^{\circ}$ boxes and based on all stars without any distance selection. The projection does not appear to be caused by any continous distribution of matter but LDN 1622 is seen to be located on the $E_{B-V}=0$. 075 edge of an extended feature.

The discontinuity at $\sim 160 \mathrm{pc}$ is not only present just in front of LDN 1622 as Fig. 7 shows for data between one and two 


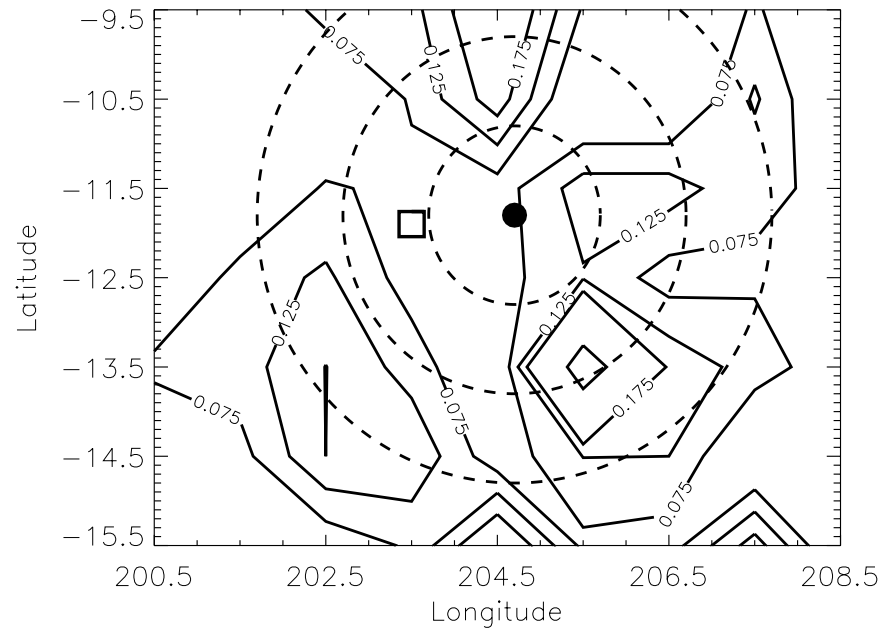

Fig. 9. Projected dust distribution based on all Hipparcos/Michigan stars selected without any $V-A_{V}-M_{V}$ criterion. Color excesses are average values in $1 \times 1 \square^{\circ}$ boxes. Dashed curves are circles with radii $1^{\circ}, 2^{\circ}$ and $3^{\circ}$ centered on the LDN 1622 position. Filled circle indicates LDN 1622 position and box that of LDN 1617.

degrees away from the cloud position. A very well defined onset of reddening is again seen at $\sim 160 \mathrm{pc}$. The projected distribution in Fig. 9 indicates several apparently isolated dust clouds in this annulus: $(l, b)=(204.5,-10.5),(205.5,-12.0$ and $(205.5,-13.5)$; two of which have the central reddening 0. 175 .

For the next one degree annulus, from 2.0 to 3.0 , the data are shown in Fig. 8. The jump at $V-A_{V}-M_{V}=6$ is much less pronounced but here another discontinuity appears at $V-A_{V}-M_{V}=7.5-8.0$ equivalent to $\approx 400 \mathrm{pc}$, which is the nominal distance previously accepted for the OB association responsible for shaping and ionizing LDN 1622 and 1617. The parallax errors for stars at $\approx 400 \mathrm{pc}$ are close to $30 \%$ but the error of the mean will be acceptable considering that the extinction jump is defined by $6-7$ stars. The $\sim 400$ pc discontinuity is not seen in the Tycho-2nMichigan data due to the upper distance limit of $\sim 400 \mathrm{pc}$ for unreddened stars discussed in the previous section. Figure 9 shows that another projected cloud appears in the 2.0 to 3.0 annulus at $(202.5,-13.5)$.

If only stars within $\sim 200$ pc are selected from their Hipparcos parallax a much more simple projected dust distribution appears as pictured in Fig. 10. Due to the positional coincidence it is tempting to associate LDN 1622 to the cloud with center $(l, b) \approx(205.5,-12.0)$ and closer than $200 \mathrm{pc}$. The central position has an average excess of 0.125 in the contour plot. The stars beyond 250 pc shown in Fig. 11 show four isolated features almost entirely avoiding the innermost $1^{\circ}$ circle. This could be an artefact brought about by massive extinction around the LDN 1622 direction and situated nearer than $\approx 200$ pc and so opaque that it pushes the apparent magnitude above the faint limit of the Hipparcos Catalogue and thus makes the extinction unmeasurable.

Comparing the absorptions measured in the two jumps, the $160 \mathrm{pc}$ one goes up to about $A_{V} \approx 0.6 \mathrm{mag}$ but $A_{V}$ reaches a full magnitude in the $\sim 400 \mathrm{pc}$ one but due to the positional coincidence in Fig. 10 the 160 pc cloud may have to be preferred

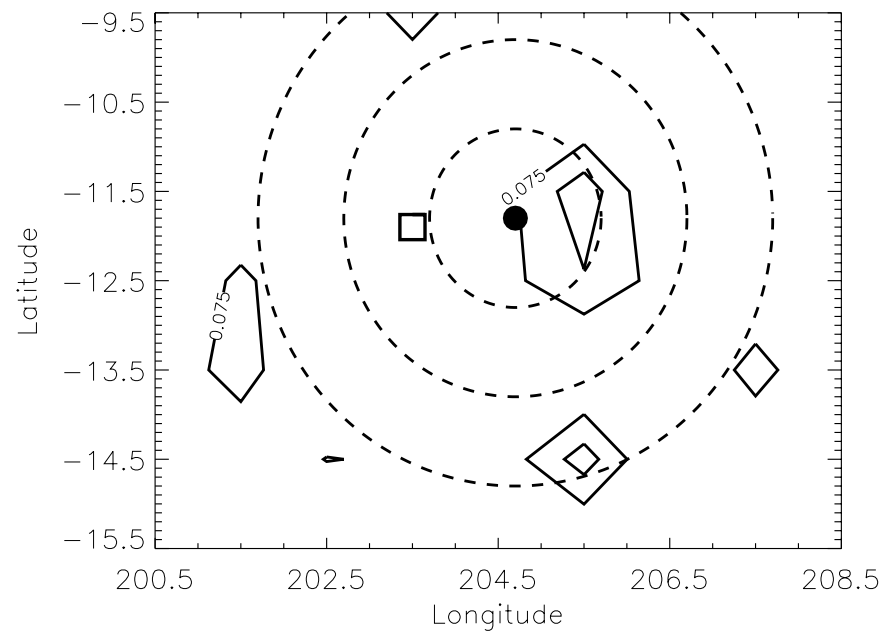

Fig. 10. Based on stars with $V-A_{V}-M_{V}$ less than $6.5(\lesssim 200 \mathrm{pc})$.

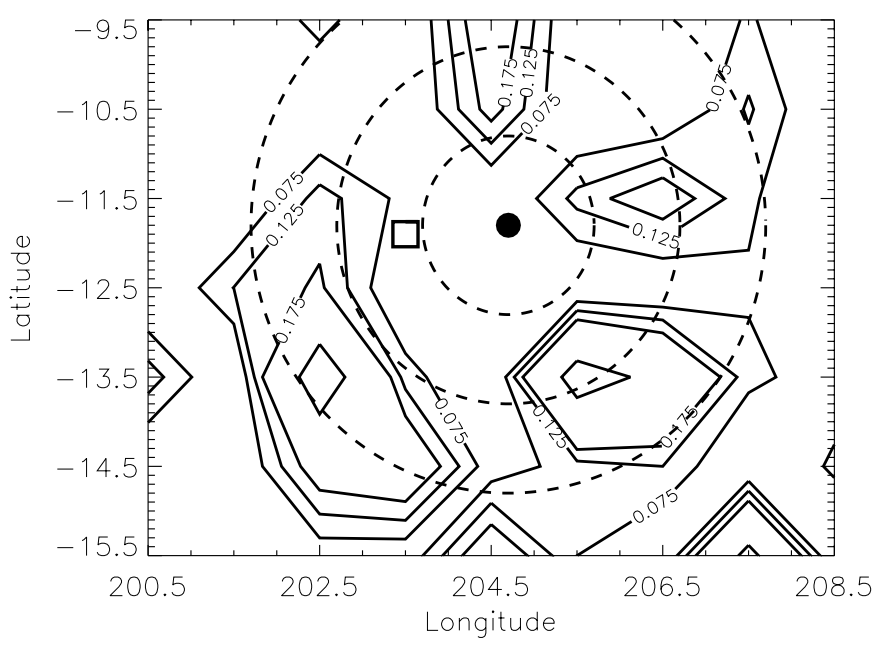

Fig. 11. Based on stars with $V-A_{V}-M_{V}$ greater than $7.0(\gtrsim 250 \mathrm{pc})$.

for a LDN 1622 identification. The first dust encountered is a bit too massive to be attributed to the diffuse interstellar clouds, so it might be associated with molecular material.

So if the reddening discontinuities are real and not just due to selection effects caused by the cut of the Hipparcos and Michigan catalogues or by the way the real interstellar extinction is distributed in space, we have two main options for the location of LDN $1622, \sim 160$ pc or $\sim 400$ pc. Only radial velocities of e.g. NaI D absorption lines observed in some of the stars defining the discontinuities similar to the $\mathrm{CO}$ velocities (Jørgensen 2000) from the LDN 1622 gas may decide this issue.

We draw attention to a remark by Bally (2001) in his discussion of the ISM structure in the Orion/Eridanus bubble just mentioning that the near side of this expanding bubble could be as close as $180 \mathrm{pc}$. This is comparable to the $160 \mathrm{pc}$ where the first extinction jump is encountered. The different radial velocities $\sim 1 \mathrm{~km} \mathrm{~s}^{-1}$ and $\sim 10 \mathrm{~km} \mathrm{~s}^{-1}$ measured for LDN 1622 and 1617 respectively (Bally 2001) might suggest that they belong to the near and far part of the expanding Orion bubble. 


\section{Conclusion}

We have calibrated the Michigan class V stars in the range from A0 to G5 in terms of intrinsic Tycho-2 color $\left(B_{\mathrm{T}}-V_{\mathrm{T}}\right)_{0}$ and absolute magnitude $M_{V_{\mathrm{T}}}$, Table 1 .

We have used this calibration to study the extinction in the direction of a minor star forming cloud LDN 1622 thought to be locally influenced by the Orion OB $1 \mathrm{~b}$ association at $\approx 450 \mathrm{pc}$. The spatial dust distribution derived from the calibration, however, shows that molecular material may be located as close as $160 \mathrm{pc}$.

There is a near positional coincidence of LDN 1622 and a projected dust cloud apparent in the color excess contours from Hipparcos/Michigan stars closer than 200 pc.

\section{References}

Allen's Astrophysical Quantities, fourth Edition, 2000, ed. A. N. Cox (Berlin: Springer Verlag)

Arce, H. G., \& Goodman, A. A. 1999, ApJ, 517, 264

Bally, J. 2001, in Tetons-4: Galactic structure, stars and the interstellar medium, ed. C. E. Woodward, M. D. Bicay, \& J. M. Shull (San Francisco: ASP), ASP Conf. Ser., 231, 204

Bessell, M. S., \& Brett, J. M. 1988, PASP, 100, 1134

Chereul, E., Crézé, M., \& Bienaymé, O. 1999, A\&AS, 135, 5

Corradi, W. J. B., Franco, G. A. P., \& Knude, J. 1998, A\&A, 326, 121

Crawford, D. 1975, AJ, 80, 955

Crawford, D. 1978, AJ, 83, 48

Crawford, D. 1979, AJ, 84, 1858

Dolan, C. J., \& Mathieu, R. D. 2001, AJ, 1212124

Domingo, A., \& Figueras, F. 1999, A\&A, 343, 446

ESA 1997, The Hipparcos Catalogue, ESA SP-1200

Fabricius, C., Makarov, V. V., Knude, J., \& Wycoff, G. L. 2002, A\&A, 386, 709
Guillout, P., Sterzik, M. F., Schmitt, J. H. M. M., Motch, C., \& Neuhäuser, R. 1998, A\&A, 337, 113

Hauck, B., \& Mermilliod, M. 1998, A\&AS, 129, 431

Hilton, J., \& Lahulla, J. F. 1995, A\&AS, 113, 325

Houk, N., \& Swift, C. 1999, Michigan Catalogue of Two-Dimensional Spectral Types for the HD Stars, vol. 5, Department of Astronomy, University of Michigan, Ann Arbor Michigan 1999

Høg, E., Fabricius, C., Makarov, V. V., et al. 2000, A\&A, 355, L27

Jordi, C., Masana, E., Luri, X., Torra, J., \& Figueras, F. 1997, in ESA Hipparcos Venice '97, ESA SP-492, 283

Jørgensen, J. 2000, Aspects of Star Formation. A study of the molecular cloud LDN 1622. Master Thesis. Niels Bohr Institute for Astrophysics, Physics and Geophysics, Copenhagen 2000

Knude, J. 1997, A\&A, 327, 90

Knude, J., \& Høg, E. 1998, A\&A, 338, 897

Knude, J., Jønch-Sørensen, H., \& Nielsen, A. S. 1999, A\&A, 350, 985

Lee, C. W., \& Myers, P. C. 1999, ApJS, 123, 233

Lejeune, J., \& Schaerer, D. 2001, A\&A, 366, 538

Nakajima, Y., Tachihara, K., Hanakawa, T., \& Nakano, M. 1998, ApJ, 497, 721

Newberg, H. J., \& Yanni, B. 1998, ApJ, 499, L57

Nielsen, A. S., Jønch-Sørensen, H., \& Knude, J. 2000, A\&A, 358, 1077

Olsen, E. H. 1984, A\&A, 57, 443

Reipurth, B., \& Olberg, M. 1991, A\&A, 246, 535

Schlegel, D. J., Finkbeiner, D. P., \& Davis, M. 1998, ApJ, 500, 525

Schmidt-Kaler, T. 1982, Landolt-Börnstein VI 2b, 1

Sfeir, D. M., Lallement, R., Crifo, F., \& Welsh, B. Y. 1999, A\&A, 346, 785

Skrutskie, M. F., Schneider, S. E., Stiening, R., et al. 1997, in The Impact of Large Scale Near-IR Sky Surveys, ed. F. Garzon, et al. (Dordrecht: Kluwer), 187

Vergely, J.-L., Freire Ferrero, R., Egret, D., \& Köppen, J. 1998, A\&A, 340, 543 